\section{Delayed Diagnosis of Esophageal Fistula Associated with Malignancy}

Sir,

Acquired connections between esophagus and respiratory tract that involve trachea, bronchi and lungs are uncommon but can become life-threatening conditions. ${ }^{1-4}$ Fistulas may have benign or malignant etiologies; bronchogenic cancer, lymphoma, chemotherapy and radiotherapy play a major role among the older people. ${ }^{1-4}$ The use of bevacizumab in cases with large subcarinal lymph nodes increases the risk for fistula. ${ }^{2}$ Aerodigestive fistulas are probably underdiagnosed and/or underreported, and the estimated low incidence seems to be misleading as postmortem studies may reveal.3,4 Whenever the diagnosis of malignancy is assumed to explain symptoms, the specific investigations to detect the fistula are not performed. Because of low index of suspicion, some cases are incidentally diagnosed during the course of respiratory assessments. ${ }^{3,4}$ The later the correct diagnosis is established, the worst will be the outcome.1,3,4

We read in this Journal the interesting case report by Kupeli and Dogan about successful treatment of latediagnosed esophageal perforation in a 60-year man. ${ }^{1}$ The esophageal tear was caused by stuck food not initially cited in anamnesis, and evolved with mediastinal and pericardial infection, as well as purulent pleural effusion. The authors performed a thoracotomy and utilised a self-expandable esophageal stent. They highlighted the history taking, esophagoscopy, and chest images; moreover, conservative management with nothing per oral route and antibiotics was emphasised. ${ }^{1}$ Hospital discharge was on day 35 , and stent withdrawal was on 71 postoperative day. Worthy of note was improvement of severe complications despite tardive diagnosis. ${ }^{1}$

In the context of malignant aerodigestive fistula not detected in vivo, comments about a 46-year Brazilian woman with lung carcinoma may be worthwhile. 4 The hallmarks of this case study were clinical and radiographic presentations mimicking a refractory excavated pneumonia by Klebsiella spp., pathogen that grew in cultures of aspirates obtained by bronchoscopy; whilst malignant cells were not observed. There was only partial response to the antibiotic therapy, and the irreversible acute respiratory distress and multiorgan failure prevented the complementary investigations. 4 Autopsy study established the diagnosis of high-grade poorly differentiated carcinoma, large cell variant with hepatic, mediastinal and subcarinal lymph node metastases. Additional findings were a cavitary tumor in the right lower lobe, which was infiltrating the esophageal wall around the site of the fistula; and metastases in the cerebral cortex. ${ }^{4}$ The authors emphasised the rarity of aerodigestive fistula as the initial presentation of a pulmonary carcinoma, which caused the death of a middle-aged never-smoker woman.

Postmortem studies often contribute to better understanding of challenging clinical pitfalls, in addition to providing solid foundations for the development of medical science as a whole. Case studies may enhance the suspicion index of health workers about rare conditions.

\section{CONFLICT OF INTEREST:}

Authors declared no conflict of interest.

\section{AUTHORS' CONTRIBUTION:}

VMDS, LAMDS: Contributed to the conception and design of the Letter; Did the acquisition, analysis, and interpretation of data for the work; drafted and revised the manuscript critically for the intellectual content; and final approval of the version to be published.

\section{REFERENCES}

1. Kupeli M, Dogan A. Successful treatment of a late diagnosed esophageal perforation with mediastinitis and pericardial abscess. J Coll Physicians Surg Pak 2018; 28:972-3.

2. Nishie K, Yasuo M, Kitaguchi Y, Kobayashi N, Tateishi K, Ushiki A, et al. Bevacizumab-induced tracheoesophageal fistula in a patient suffering from lung cancer with bulky subcarinal lymph node: A case report. Nagoya J Med Sci 2018; 80:129-34.

3. Qureshi YA, Muntzer Mughal M, Fragkos KC, Lawrence D, George J, Mohammadi B, et al. Acquired adult aerodigestive fistula: Classification and management. J Gastrointest Surg 2018; 22:1785-94.

4. dos Santos VM, dos Santos LAM. Esophageal pulmonary fistula associated with lung cancer - autopsy study. Neumol Cir Torax 2018; 77:293-6.

Vitorino Modesto dos Santos ${ }^{1}$ and Lister Arruda Modesto dos Santos $^{2}$

1 Department of Internal Medicine, Armed Forces Hospital and Catholic University, Brasilia-DF, Brazil

2 Department of General Surgery, State Workers Hospital, São Paulo-SP (HSE), Brazil

Correspondence to: Prof. Vitorino Modesto dos Santos, Armed Forces Hospital, Estrada do Contorno do Bosque s/n, Cruzeiro Novo, CEP 70658-900. Brasília-DF, Brazil

E-mail: vitorinomodesto@gmail.com

Received: January 29, 2019; Revised: February 26, 2019;

Accepted: February 26, 2019.

$$
\cdots \cdot \hat{n} \cdot \ldots
$$

